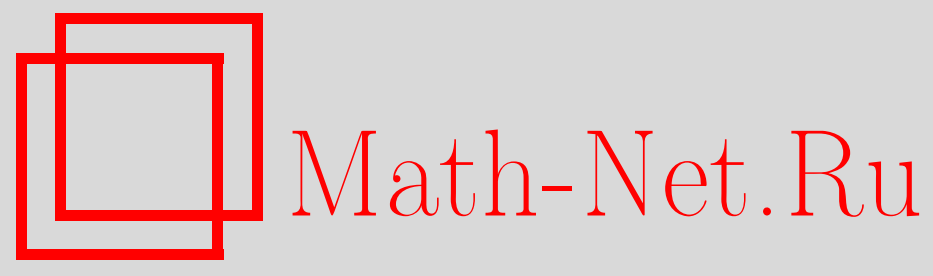

T. В. Андреева, Об одном обобщении метода граничных функционалов, Дискрет. матем., 2004, том 16, выпуск 3, 85-104

DOI: https://doi.org/10.4213/dm165

Использование Общероссийского математического портала Math-Net.Ru подразумевает, что вы прочитали и согласны с пользовательским соглашением http://www . mathnet.ru/rus/agreement

Параметры загрузки:

IP : 18.234 .197 .8

26 апреля 2023 г., 18:16:48

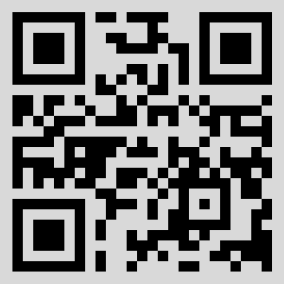


УдК 519.1

\title{
Об одном обобщении метода граничных функционалов
}

\author{
() 2004 г. T. В. Андреева
}

Метод граничных функционалов был предложен А. А. Сапоженко для решения ряда перечислительных задач. В данной статье метод обобщен на случай функциональных пар с характеристикой 3 и получена асимптотическая формула для сумм граничных функционалов по регуліяным семействам подмножеств.

Работа выполнена при поддержке Российского фонда фундаментальных исследований, проект 04-01-00359.

\section{1. Введение}

А. А. Сапоженко (см. [1, 2]) предложил метод граничных функционалов для решения перечислительных задач. Метод применим в случае, когда перечислительная задача сводится к вычислению сумм вида

$$
T(X, f)=\sum_{A \subseteq X} f(A)
$$

где $f$ - граничный функционал (см. раздел 1). Пусть $\mathscr{A}(X, f)$ - семейство всех связных подмножеств множества $X$. Тогда для всякого $\mathscr{B} \subseteq A(X, f)$ обозначим через $C(\mathscr{B})$ семейство множеств $A \subseteq X$ таких, что каждая компонента связности множества $A$ принадлежит семейству $\mathscr{B}$. Положим

$$
S(\mathscr{B})=\sum_{B \in C(\mathscr{B})} f(B)
$$

Поскольку

$$
T(X, f)=S(\mathscr{A}(X, f)),
$$

задача вычисления сумм $T(X, f)$ сводится к аналогичной задаче для сумм вида $S(\mathscr{B})$, где $\mathscr{B} \subseteq A(X, f)$. Идея метода состоит в том, что суммы вида (1) выражаются через достаточно просто вычисляемые суммы вида

$$
\alpha^{\nu}(\mathscr{B})=\sum_{B \in \mathscr{B}}(f(B))^{\nu}
$$


Метод граничных функционалов представляет собой аппарат для получения асимптотических решений перечислительных задач таких, как нахождение асимптотики числа антицепей в различных частично унорядоченных множествах.

В [2] А. А. Сапоженко предложил решение проблемы Дедекинда о числе монотонных булевых функций на основе метода граничных функщионалов. При этом рассматриваемые семейства связных множеств $\mathscr{B}$ оказались регулярными (см. раздел 5) и такими, что мощность всякого множества из $\mathscr{B}$ не превосходила $\Delta=2$. В данной работе получена асимптотика сумм вида 1 для почти регулярных семейств подмножеств (см. раздел 5) при $\Delta=3$.

Основным результатом работы является следующее утверждение.

Теорема 1. Пусть $I=(X, f)$ является $(3, x, q, c)$-ординарной функциональной парой, $\mathscr{B} \subseteq \mathscr{A}_{\hat{3}}(I)$ является почти регулярным семейством. Тогда

$$
S(\mathscr{B})=\left(1+O\left(2^{-(1 / 2) \log ^{2} x}\right)\right) \exp \left\{\hat{\mu}_{3}(\mathscr{B})\right\}
$$

где

$$
\hat{\mu}_{3}(\mathscr{B})=\alpha^{1}(\mathscr{B})-\frac{1}{2} \alpha^{2}(\mathscr{B})+\frac{1}{3} \alpha^{3}(\mathscr{B})-\alpha^{1}\left(\mathscr{B}_{[2,3]}^{(2)}\right)+\alpha^{1}\left(\mathscr{B}_{[3]}^{(1,2)}\right)
$$

Доказательство теоремы приведено в разделе 6.

\section{2. Определения и известные результаты}

Для полноты изложения в этом разделе будут приведены определения и результаты из статьи [4]. Кроме того, будут получены оценки сумм $\tilde{S}(\mathscr{B})$, аналогичные оценкам сумм $S(\mathscr{B})$ (следствие 1, теорема 4).

Если $X$ - множество, то через $2^{X}$ обозначается семейство всех его подмножеств. Произвольное отображение $f: 2^{X} \rightarrow(0,1]$ называется граничным функционалом на семействе подмножеств множества $X$, если оно удовлетворяет следующим трем условиям:

1) $f(A)=1$ тогда и только тогда, когда $A=\varnothing$;

2) $f(A \cup B) \geqslant f(A) f(B)$;

3) $f(A \cup B)>f(A) f(B)$ тогда и только тогда, когда существуют $u \in A$ и $v \in B$ такие, что $f(\{u, v\})>f(\{u\}) f(\{v\})$.

Пара $I=(X, f)$, где $X$ - конечное множество, а $f$ - граничный функционал, называется функциональной парой.

Пусть $I=(X, f)$ - функциональная пара, введем понятие множества (семейства) ранга $r$ над $X$. Произвольное подмножество $A \subseteq X$ называется семейством ранга 1 . Если $A_{1}, \ldots, A_{s}$ - различные семейства ранга $r$, то $F=\left\{A_{1}, \ldots, A_{s}\right\}$ называется семейством ранга $r+1$. Семейства $A_{1}, \ldots, A_{s}$ называются элементами семейства $F$. Множество всех семейств ранга $r$ над $X$ обозначается через $X^{(r)}$.

Для произвольного семейства $F$ ранга $r$ по индукщия определены величины $|F|-$ мощность семейства $F,\|F\|$ - мультимощность семейства $F, f(F)$ - значение функщионала на семействе $F$ и множество $\langle F\rangle$ - основа семейства $F$. Если $F$-семейство ранга 1 , то есть $F \subseteq X$, то $\|F\|=|F|$, где $|F|$ - число элементов множества $F, f(F)$ определяется 
функщиональной парой $I=(X, f)$, а $\langle F\rangle=F$. Пусть для некоторого $r \geqslant 1$ эти величины и множество определены, и пусть $F=\left\{A_{1}, \ldots, A_{s}\right\}$ - семейство ранга $r+1$. Тогда

$$
|F|=s, \quad\|F\|=\sum_{1 \leqslant i \leqslant s}\left\|A_{i}\right\|, \quad f(F)=\prod_{1 \leqslant i \leqslant s} f\left(A_{i}\right), \quad\langle F\rangle=\bigcup_{1 \leqslant i \leqslant s}\left\langle A_{i}\right\rangle .
$$

Пусть $I=(X, f)-$ функциональная пара. Вершины $u$ и $v$ из $X$ такие, что $f(\{u, v\})>f(\{u\}) f(\{v\})$, называются смежными (используется обозначение $u$ \# $v)$. Вершины $u$ и $v$, для которых выполнено равенство $f(\{u, v\})=f(\{u\}) f(\{v\})$, называются несмежными (используется обозначение $u \| v$ ). Множества $A \subseteq X$ и $B \subseteq X$ называются смежными (используется обозначение $A \# B$ ), если $f(A \cup B)>f(A) f(B)$. Множества $A$ и $B$ называются несмежными (используется обозначение $A \| B$ ), если $f(A \cup B)=f(A) f(B)$.

Граф $G_{l}=(X ; E)$ с множеством ребер $E=\{\{u, v\}: u$ \# $v\}$ называется графом функциональной пары $I=(X, f)$. Пусть $A \subseteq X, E^{\prime}=\{\{u, v\} \in E:\{u, v\} \subseteq A\}$. Через $H_{G_{I}}(A)=\left(A ; E^{\prime}\right)$ обозначается подграф графа $G_{I}$, порожденный множеством $A$.

Введем понятие связного семейства ранга $r$. Пусть $I=(X, f)-$ функциональная пара. Для семейств ранга 1 связность определяется с помощью графа $G_{I}$ функциональной пары $I$. Именно, непустое множество $F \subseteq X$ называется связным семейством ранга 1 , если подграф $H_{G_{I}}(F)$ графа $G_{I}$, порожденный множеством $F$, связен.

Семейство $F=\left\{A_{1}, \ldots, A_{s}\right\}$ ранга $r>1$ называется связным, если каждое из $A_{i}$, $i=1, \ldots, s$, является связным семейством ранга, не превышающего $r-1$, причем существует семейство $A_{i}$ ранга $r-1$, и $\langle F\rangle \in \mathscr{A}(I)$. Множество всех связных семейств ранга $r$ обозначается через $\mathscr{A}^{(r)}(I)$. По определению $\mathscr{A}^{(1)}(I)=\mathscr{A}(I)$.

Для произвольного подсемейства $\mathscr{B} \subseteq \mathscr{A}(I)$ определено семейство $\mathscr{B}^{(r)}$ связных подмножеств ранга $r$, порожденное множеством $\mathscr{B}$. Положим $\mathscr{B}^{(1)}=\mathscr{B}$. Если $\mathscr{B}^{(r-1)}$ определено, то

$$
\mathscr{B}^{(r)}=2^{\mathscr{B}^{(r-1)}} \cap \mathscr{A}^{(r)}(I) .
$$

Пусть $A \subseteq X$, множество $B \subseteq A$ называется компонентой связности множества $A$, если граф $H_{G_{I}}(B)$ является компонентой связности графа $H_{G_{I}}(A)$. Подсемейство $B$ семейства $F$ ранга $r$ называется компонентой связности семейства $F$ (используется обозначение $B \vdash F$ ), если $B \in \mathscr{A}^{(r)}(I)$ и $\langle B\rangle-$ компонента связности множества $\langle F\rangle$. Через $\eta(F)$ обозначается число компонент связности семейства $F$. Для функщиональной пары $I$ и натуральных $j, k$

$$
\mathscr{A}_{j}(I)=\{A \in \mathscr{A}(I):|A|=j\}, \quad \mathscr{A}_{\hat{k}}(I)=\bigcup_{j \leqslant k} \mathscr{A}_{j}(I) .
$$

Для натуральных $r, j$ и $\mathscr{B} \subseteq X^{(r)}$

$$
\mathscr{B}_{[j]}=\{B \in \mathscr{B}:\|B\|=j\}
$$

Если $\mathscr{B} \subseteq \mathscr{A}^{(r)}(I)$, то

$$
C(\mathscr{B})=\left\{F \subseteq X^{(r)}: A \vdash F \Rightarrow A \in \mathscr{B}\right\}, \quad C_{j}(\mathscr{B})=\{F \in C(\mathscr{B}): \eta(F)=j\} .
$$

Если $\mathscr{B} \subseteq X^{(r)}, B \in X^{(s)}, r, s \geqslant 1$, то

$$
\mathscr{B}_{B}=\{A \in \mathscr{B}:\langle A\rangle \#\langle B\rangle\}, \quad \mathscr{B}_{\bar{B}}=\{A \in \mathscr{B}:\langle A\rangle \|\langle B\rangle\} .
$$


Введем некоторые суммы граничных функщионалов. Пусть $\nu, r, \varkappa-$ натуральные числа и $\mathscr{B} \subseteq \mathscr{A}(I)-$ произвольное семейство, тогда

$$
\alpha^{\nu}\left(\mathscr{B}^{(r)}\right)=\sum_{A \in \mathscr{B}(r)} f^{\nu}(A), \quad S(\mathscr{B})=\sum_{A \in C(\mathscr{B})} f(A), \quad S_{k}(\mathscr{B})=\sum_{A \in C_{k}(\mathscr{B})} f(A),
$$

здесь и далее $f^{\nu}(A)=(f(A))^{\nu}$.

Приведем некоторые соотношения между суммами $\alpha^{\nu}\left(\mathscr{B}^{(r)}\right)$ и $S(\mathscr{B})$, доказанные в [4]. Пусть $\mathscr{D}=B^{(r-1)}$, тогда через $\mathscr{D}^{\prime}$ обозначим семейство $\mathscr{B}^{(r)}$.

Лемма 1. Для любой функциональной пары $I=(X, f)$, любого натурального числа $r$ $u$ любого $\mathscr{B} \subseteq \mathscr{A}^{(r)}(I)$

$$
\prod_{A \in \mathscr{B}}(1+f(A))=\sum_{B \in C(\mathscr{B})} f(B) \sum_{D \in C\left(\mathscr{B}_{\bar{B}}^{\prime}\right)} f(D) .
$$

Теорема 4.1 из [4] легко переносится на случай семейства ранга $r \geqslant 1$.

Теорема 2. Пусть $r$ - натуральное число, функииональная пара $I=(X, f)$ u подсемейство $\mathscr{B} \subseteq \mathscr{A}^{(r)}(I)$ таковы, что для некоторьх положстельных $\psi$ и $\delta$

$$
\begin{gathered}
\alpha^{1}\left(\mathscr{B}^{\prime}\right) \leqslant \psi, \\
\alpha^{2}(\mathscr{B}) \leqslant \delta .
\end{gathered}
$$

Tогдa

$$
\exp \left\{\alpha^{1}(\mathscr{B})-\psi-\delta / 2\right\} \leqslant S(\mathscr{B}) \leqslant \exp \left\{\alpha^{1}(\mathscr{B})\right\} .
$$

Теорема 3. Пусть $I=(X, f)-$ функчиональная пара, подсемейство $\mathscr{B} \subseteq \mathscr{A}(I)$ и положительные числа $\lambda$ и $\gamma$ таковы, что

$$
\begin{aligned}
& \alpha^{1}\left(\mathscr{B}^{(3)}\right) \leqslant \lambda, \\
& \alpha^{2}\left(\mathscr{B}^{(2)}\right) \leqslant \zeta .
\end{aligned}
$$

Тогда

$$
\begin{aligned}
\exp \left\{-\alpha^{1}\left(\mathscr{B}^{(2)}\right)\right\} \prod_{A \in \mathscr{B}}(1+f(A)) & \leqslant \sum_{B \in C(\mathscr{B})} f(B) \exp \left\{-\alpha^{1}\left(\mathscr{B}_{B}^{(2)}\right)\right\} \\
& \leqslant \exp \left\{-\alpha^{1}\left(\mathscr{B}^{(2)}\right)+\lambda+\zeta / 2\right\} \prod_{A \in \mathscr{B}}(1+f(A)) .
\end{aligned}
$$

Доказательство. В силу леммы 1

$$
\prod_{A \in \mathscr{B}}(1+f(A))=\sum_{B \in C(\mathscr{B})} f(B) \sum_{D \in C\left(\mathscr{B}_{\bar{B}}^{(2)}\right)} f(D) .
$$

Поскольку $\mathscr{B}_{\bar{B}}^{(2)} \subseteq \mathscr{B}^{(2)}$, из теоремы 2 при $r=2, \psi=\lambda$ и $\delta=\zeta$ следует, что

$$
\exp \left\{\alpha^{1}\left(\mathscr{B}_{\bar{B}}^{(2)}\right)-\lambda-\zeta / 2\right\} \leqslant \sum_{D \in C\left(\mathscr{B}_{\bar{B}}^{(2)}\right)} f(D) \leqslant \exp \left\{\alpha^{1}\left(\mathscr{B}_{\bar{B}}^{(2)}\right)\right\} .
$$

Заметим что

$$
\alpha^{1}\left(\mathscr{B}_{\bar{B}}^{(2)}\right)=\alpha^{1}\left(\mathscr{B}^{(2)}\right)-\alpha^{1}\left(\mathscr{B}_{B}^{(2)}\right) .
$$

Теперь из (7)-(9) следует утверждение теоремы. 
Для $B \subseteq X$ положим $\pi(B)=\exp \left\{-\alpha^{1}\left(\mathscr{B}_{B}^{(2)}\right)\right\}$ и

$$
\tilde{S}(\mathscr{B})=\sum_{B \in C(\mathscr{B})} f(B) \pi(B), \quad \tilde{S}_{k}(\mathscr{B})=\sum_{B \in C_{k}(\mathscr{B})} f(B) \pi(B) .
$$

В данной работе будет получена асимптотика сумм $\tilde{S}(\mathscr{B})$ при выполнении условий (5), (6).

Лемма 2. Пусть $I=(X, f)-$ функциональная пара, $\mathscr{B} \subseteq \mathscr{A}(I), B, D \subseteq X$. Тогда

$$
\pi(B) \pi(D) \leqslant \pi(B \cup D) \leqslant \pi(B) .
$$

Доказательство. Утверждение следует из определения $\pi(B)$ и из того, что

$$
\alpha^{1}\left(\mathscr{B}_{B}^{(2)}\right) \leqslant \alpha^{1}\left(\mathscr{B}_{B \cup D}^{(2)}\right)=\alpha^{1}\left(\mathscr{B}_{B}^{(2)}\right)+\alpha^{1}\left(\mathscr{B}_{D}^{(2)}\right)-\alpha^{1}\left(\mathscr{B}_{B}^{(2)} \cap \mathscr{B}_{D}^{(2)}\right) \leqslant \alpha^{1}\left(\mathscr{B}_{B}^{(2)}\right)+\alpha^{1}\left(\mathscr{B}_{D}^{(2)}\right) \text {. }
$$

Лемма доказана.

Следствие 1. Пусть для функчиональной пары $I=(X, f)$ и подсемейства $\mathscr{B} \subseteq \mathscr{A}(I)$ выполнены условия теоремы 2, тогда

$$
\exp \left\{\alpha^{1}(\mathscr{B})-2 \psi-\delta / 2\right\} \leqslant \tilde{S}(\mathscr{B}) \leqslant \exp \left\{\alpha^{1}(\mathscr{B})\right\}
$$

Доказательство. Утверждение следует из теоремы 2 при $r=1$ и того, что

$$
S(\mathscr{B}) \exp \left\{-\alpha^{1}\left(\mathscr{B}^{(2)}\right)\right\} \leqslant \tilde{S}(\mathscr{B}) \leqslant S(\mathscr{B}) .
$$

Теорема 4. Пусть даны функциональная пара $I=(X, f)$ и подсемейство $\mathscr{B} \subseteq \mathscr{A}(I)$. Пусть $\alpha^{1}(\mathscr{B})$ достаточно велико, для положительных $\lambda$ и $\zeta$ выполняются неравенства (5) $u$ (6) $и$, кроме того, для положительного $\xi$

$$
\alpha^{4}(\mathscr{B}) \leqslant \xi
$$

Тогда

$$
\exp \{\mu(\mathscr{B})-\xi / 4\} \leqslant \tilde{S}(\mathscr{B}) \leqslant \exp \{\mu(\mathscr{B})+\lambda+\zeta / 2\}
$$

гдe

$$
\mu(\mathscr{B})=\alpha^{1}(\mathscr{B})-\frac{1}{2} \alpha^{2}(\mathscr{B})+\frac{1}{3} \alpha^{3}(\mathscr{B})-\alpha^{1}\left(\mathscr{B}^{(2)}\right) .
$$

Доказательство. С использованием неравенства

$$
\ln (1+x) \geqslant x-\frac{x^{2}}{2}+\frac{x^{3}}{3}-\frac{x^{4}}{4},
$$

справедливого при $x>0$, и условия (12) получаем, что

$$
\begin{aligned}
\prod_{A \in \mathscr{B}}(1+f(A)) & =\exp \left\{\sum_{A \in \mathscr{B}} \ln (1+f(A))\right\} \\
& \geqslant \exp \left\{\sum_{A \in \mathscr{B}}\left(f(A)-\frac{1}{2} f^{2}(A)+\frac{1}{3} f^{3}(A)-\frac{1}{4} f^{4}(A)\right)\right\} \\
& =\exp \left\{\alpha^{1}(\mathscr{B})-\frac{1}{2} \alpha^{2}(\mathscr{B})+\frac{1}{3} \alpha^{3}(\mathscr{B})-\frac{1}{4} \alpha^{4}(\mathscr{B})\right\} \\
& \geqslant \exp \left\{\alpha^{1}(\mathscr{B})-\frac{1}{2} \alpha^{2}(\mathscr{B})+\frac{1}{3} \alpha^{3}(\mathscr{B})-\frac{\xi}{4}\right\} .
\end{aligned}
$$


С другой стороны, из неравенства

$$
x-\frac{x^{2}}{2}+\frac{x^{3}}{3} \geq \ln (1+x),
$$

справедливого при $x>0$, следует, что

$$
\exp \left\{\alpha^{1}(\mathscr{B})-\frac{1}{2} \alpha^{2}(\mathscr{B})+\frac{1}{3} \alpha^{3}(\mathscr{B})\right\} \geqslant \prod_{A \in \mathscr{B}}(1+f(A)) .
$$

Из (14), (15) и теоремы 3 вытекает (13).

\section{3. Асимптотики $\tilde{S}(\mathscr{B})$ для ординарных функциональных пар}

В [4] доказано, что условия теоремы 2 автоматически выполняются для ординарных функциональных пар (определение см. ниже). Там же получены асимптотики сумм $S(\mathscr{B})$ при $\Delta \leqslant 2$. В данном разделе эти результаты обобщаются на случай сумм $\tilde{S}(\mathscr{B})$ при $\Delta=3$. Везде в дальнейшем $\Delta, x, q$ - натуральные числа, $c \geqslant 1, \varkappa$ достаточно велико, $\log a=\log _{2} a$.

Функщиональная пара $I=(X, f)$ называется $(\Delta, \varkappa, q, c)$-ординарной, если

(1) справедливо неравенство

$$
|X| \leqslant 2^{(\Delta+1) x-2 \log ^{2} x},
$$

(2) для всякого $v \in X$

$$
f(\{v\}) \leqslant 2^{-x}
$$

(3) для любых $A \subseteq X$ и $v \in A$

$$
f(A) \leqslant f(A \backslash\{v\}) f(\{v\}) 2^{(|A|-1) q},
$$

(4) для любого $v \in X$ и любого натурального числа $m$

$$
\left|\mathbb{A}_{\{v\}, \hat{m}}(I)\right| \leqslant x^{c m}
$$

Если какие-либо из свойств (16)-(19) не выполняются для функциональной пары $I=(X, f)$, в соответствующих координатах ставятся прочерки. Например, если функциональная пара $I=(X, f)$ удовлетворяет свойствам (17) и (18), но, быть может, не удовлетворяет свойствам (16), (19), то такая пара называется $(-, \varkappa, q,-)$-ординарной.

В [4] доказаны следующие утверждения.

Лемма 3. Пусть $I=(X, f)$ является $(-, x, q,-)$-ординарной функциональной парой, $\mathscr{B} \subseteq \mathscr{A}_{\hat{m}}(I) u A \in C(\mathscr{B})$. Тогдa

$$
f(A) \leqslant 2^{-|A|(x-q(m-1) / 2)} .
$$


Лемма 4. Пусть $I=(X, f)$ является $(-, \varkappa, q, c)$-ординарной функииональной парой, $B \subseteq X u \mathscr{B} \subseteq \mathscr{A}_{\hat{m}}(I)$. Тогда для всех натуральных $v, r, j$

$$
\alpha^{\nu}\left(\mathscr{B}_{B,[j]}^{(r)}\right) \leqslant|B| 8^{(r-1) j} \varkappa^{c r j} 2^{-j(\varkappa-q(m-1) / 2) \nu} .
$$

Следствие 2. Пусть $I=(X, f)$ является $(\Delta, x, q, c)$-ординарной функииональной парой, $\nu, r, j$ - произвольные фиксированные натуральные числа, $\mathscr{B} \subseteq \mathscr{A}_{\hat{m}}(I), m \leqslant\lceil\varkappa /(2 q)\rceil, a$ $\varkappa$ достаточно велико. Тогда

$$
\alpha^{\nu}\left(\mathscr{B}_{[j]}^{(r)}\right) \leqslant 2^{-(v j-(\Delta+1)) x-(3 / 2) \log ^{2} x}
$$

$n p u j \leqslant(\log \varkappa) /(3 c r) u$

$$
\alpha^{\nu}\left(\mathscr{B}_{[j]}^{(r)}\right) \leqslant 2^{-\nu x j / 2}
$$

npu $j>(\log \varkappa) /(3 c r)$.

Докажем результаты для $\tilde{S}(\mathscr{B})$, аналогичные результатам для $S(\mathscr{B})$.

Лемма 5. Пусть $I=(X, f)$ является $(\Delta, \varkappa, q, c)$-ординарной функциональной парой, $r_{0}=\lceil\varkappa /(2 q)\rceil, \mathscr{B} \subseteq \mathscr{A}_{\hat{r}_{0}}(I)$ и $\varkappa$ достаточно велико. Тогда

$$
\tilde{S}(\mathscr{B})=\left(1+O\left(2^{-2 \log ^{2} x}\right)\right) \tilde{S}\left(\mathscr{B} \cap \mathscr{A}_{\hat{\Delta}}(I)\right) .
$$

Доказательство. Положим $\widehat{\mathscr{B}}=\mathscr{B} \cap \mathscr{A}_{\hat{\Delta}}(I)$ и $\overline{\mathscr{B}}=\mathscr{B} \backslash \widehat{\mathscr{B}}$. С использованием (10), (22) и (23) с $r=1, v=1$ и теоремы 2 находим, что

$$
\begin{aligned}
\tilde{S}(\widehat{\mathscr{B}}) & \leqslant \tilde{S}(\mathscr{B})=\tilde{S}(\hat{B} \cup \overline{\mathscr{B}})=\sum_{B \in C(\widehat{\mathscr{B}})} f(B) \sum_{D \in C\left(\overline{\mathscr{B}}_{\bar{B}}\right)} f(D) \pi(B \cup D) \\
& \leqslant \sum_{B \in C(\widehat{\mathscr{P}})} f(B) \pi(B) \sum_{D \in C(\overline{\mathscr{B}})} f(D) \leqslant \tilde{S}(\widehat{\mathscr{B}}) \exp \left\{\alpha^{1}(\overline{\mathscr{B}})\right\} \leqslant\left(1+O\left(2^{-2 \log ^{2} x}\right)\right) \tilde{S}(\widehat{\mathscr{B}}) .
\end{aligned}
$$

Теорема 5. Пусть $I=(X, f)$ является $(3, \varkappa, q, c)$-ординарной функциональной парой, $\mathscr{B} \subseteq \mathscr{A}_{\hat{3}}(I)$. Тогда при достаточно больших $\varkappa$

$$
\tilde{S}(\mathscr{B})=\left(1+O\left(2^{-\log ^{2} x}\right)\right) \exp \{\mu(\mathscr{B})\}
$$

где

$$
\mu(\mathscr{B})=\alpha^{1}(\mathscr{B})-\frac{1}{2} \alpha^{2}(\mathscr{B})+\frac{1}{3} \alpha^{3}(\mathscr{B})-\alpha^{1}\left(\mathscr{B}^{(2)}\right) .
$$

Доказательство. Предположим сначала, что

$$
\alpha^{1}(\mathscr{B}) \geqslant \varkappa,
$$

и следовательно, $\alpha^{1}(\mathscr{B})$ достаточно велико. Воспользуемся теоремой 4 . Для этого докажем, что условия (5), (6) и (12) выполнены соответственно при $\lambda=\zeta=2^{-\log ^{2} x}$ и $\xi=2^{-(3 / 2) \log ^{2} x}$. Тогда (25) будет следовать из (13).

Проверим выполнение условия (5). Положим $j_{0}=(\log x) /(3 c r)$. В силу (22) и (23) с $r=3$ при достаточно больших $\varkappa$

$$
\alpha^{1}\left(\mathscr{B}^{(3)}\right)=\sum_{j \geqslant 4} \alpha^{1}\left(\mathscr{B}_{[j]}^{(3)}\right) \leqslant \sum_{4 \leqslant j \leqslant j_{0}} 2^{-(j-4) x-(3 / 2) \log ^{2} x}+\sum_{j>j_{0}} 2^{-j x / 2} \leqslant 2^{-\log ^{2} x} .
$$


Отсюда вытекает (19) с $\lambda=2^{-\log ^{2} x}$.

Докажем выполнение условия (6) при $\zeta=2^{-\log ^{2} x}$. В силу (22) и (23) при $v=2$, $r=2, \Delta=3$

$$
\alpha^{2}\left(\mathscr{B}^{(2)}\right)=\sum_{j \geqslant 2} \alpha^{2}\left(\mathscr{B}_{[j]}^{(2)}\right) \leqslant \sum_{2 \leqslant j \leqslant j_{0}} 2^{-(2 j-4) x-(3 / 2) \log ^{2} x}+\sum_{j>j_{0}} 2^{-j x} \leqslant 2^{-\log ^{2} x} .
$$

Докажем выполнение условия (12). Оценим $\alpha^{1}(\mathscr{B})$ сверху. Заметим, что в силу (16)(18) и (19) с $m=1$ и $\Delta=3$

$$
\begin{aligned}
\alpha^{1}(\mathscr{B}) & =\sum_{A \in \mathscr{B}} f(A) \leqslant \sum_{A \in \mathbb{A}_{\hat{3}}(I)} f(A)=\sum_{A \in \mathbb{A}_{1}(I)} f(A)+\sum_{A \in \mathbb{A}_{2}(I)} f(A)+\sum_{A \in \mathscr{A}_{3}(I)} f(A) \\
& \leqslant \sum_{v \in X} f(\{v\})+\sum_{v \in X} \sum_{\{u\} \#\{v\}} f(\{u, v\})+\sum_{v \in X} \sum_{\{u, w\} \#\{v\}} f(\{u, v, w\}) \\
& \leqslant|X| 2^{-x}+|X| x^{c} 2^{-2 x+q}+|X| x^{2 c} 2^{-3 x+2 q} \\
& \leqslant 2^{3 x-2 \log ^{2} x}+\varkappa^{c} 2^{2 x+q}+\varkappa^{2 c} 2^{x+2 q} \leqslant 2^{3 x-(3 / 2) \log ^{2} x} .
\end{aligned}
$$

С использованием (29) и (17) при $\Delta=3$ находим, что

$$
\alpha^{4}(\mathscr{B}) \leqslant \alpha^{1}(\mathscr{B}) 2^{-3 x} \leqslant 2^{-3 x} 2^{3 x-(3 / 2) \log ^{2} x} \leqslant 2^{-(3 / 2) \log ^{2} x} .
$$

Отсюда следует (12) с $\xi=2^{-(3 / 2) \log ^{2} x}$. Таким образом, условия теоремы 4 выполнены при указанных значениях параметров $\lambda, \zeta$ и $\xi$. Теперь из (13), (27)-(30) вытекает (25), а значит, утверждение доказано при условии (26).

Пусть теперь

$$
\alpha^{1}(\mathscr{B})<\varkappa .
$$

Покажем, что тогда выполнены условия следствия 1 при соответствующих $\psi$ и $\delta$, и (25) будет следовать из (11). Проверим выполнение условия (3). Заметим, что в силу (17) и (31) при достаточно больших $x$

$$
\alpha^{1}\left(\mathscr{B}_{[2]}^{(2)}\right)=\sum_{\{u\} \in \mathscr{B}} f(\{u\}) \sum_{\{v\} \in \mathscr{B}:\{v\} \#\{u\}} f(\{v\}) \leqslant \alpha^{1}(\mathscr{B}) x^{c} 2^{-\varkappa} \leqslant \varkappa^{c+1} 2^{-\varkappa} .
$$

Аналогично с использованием (17) и (18) получаем, что

$$
\begin{aligned}
& \alpha^{1}\left(\mathscr{B}_{[3]}^{(2)}\right) \leqslant\left(2^{q}+1\right) \varkappa^{2 c+1} 2^{-2 \varkappa}, \\
& \alpha^{1}\left(\mathscr{B}_{[4]}^{(2)}\right) \leqslant 3 \cdot 2^{q} \varkappa^{3 c+1} 2^{-3 \varkappa} .
\end{aligned}
$$

Оценивая $\alpha^{1}\left(\mathscr{B}_{[j]}^{(2)}\right)$ при $j>4$ с помощью (22) и (23), с учетом (32)-(34) находим, что

$$
\begin{aligned}
\alpha^{1}\left(\mathscr{B}^{(2)}\right)=\sum_{j \geqslant 2} \alpha^{1}\left(\mathscr{B}_{[j]}^{(2)}\right) \leqslant & \varkappa^{c+1} 2^{-\varkappa}+\left(2^{q}+1\right) \varkappa^{2 c+1} 2^{-2 x}+3 \cdot 2^{q} \varkappa^{3 c+1} 2^{-3 x} \\
& +\sum_{5 \leqslant j \leqslant j_{0}} 2^{-(j-4) x-(3 / 2) \log ^{2} \varkappa}+\sum_{j>j_{0}} 2^{-j \varkappa / 2} \leqslant 2 \cdot 2^{-\varkappa} \varkappa^{c+1} .
\end{aligned}
$$


Отсюда вытекает (3) с $\psi=2 \cdot 2^{-\varkappa} \varkappa^{c+1}$.

Проверим выполнение условия (4). Для любого $A \in \mathscr{A}_{\hat{3}}(I)$ из (20) следует, что

$$
f(A) \leqslant 2^{-|A|(x-q)} \leqslant 2^{-x+q},
$$

поэтому

$$
f^{2}(A) \leqslant f(A) 2^{-x+q} .
$$

С учетом (31) получаем, что

$$
\alpha^{2}(\mathscr{B})=\sum_{A \in \mathscr{B}} f^{2}(A) \leqslant \alpha^{1}(\mathscr{B}) 2^{-\varkappa+q} \leqslant \varkappa 2^{-\varkappa+q} .
$$

Отсюда вытекает справедливость условия (4) с $\delta=\varkappa 2^{-x+q}$. В силу (11) с учетом (3), (4) и (35) получаем, что

$$
\begin{aligned}
\tilde{S}(\mathscr{B}) & =\left(1+O\left(2^{-x} \varkappa^{c+1}\right)\right) \exp \left\{\alpha^{1}(\mathscr{B})\right\} \\
& =\left(1+O\left(2^{-\varkappa} \varkappa^{c+1}\right)\right) \exp \left\{\alpha^{1}(\mathscr{B})-\frac{1}{2} \alpha^{2}(\mathscr{B})+\frac{1}{3} \alpha^{3}(\mathscr{B})-\alpha^{1}\left(\mathscr{B}^{(2)}\right)\right\} .
\end{aligned}
$$

Утверждение доказано при условии (31).

Замечание 1. Из доказательства теоремы ясно, что при $\alpha^{1}(\mathscr{B})<\varkappa$ выполнены условия теоремы 2 с $r=1$, следовательно,

$$
S(\mathscr{B})=\left(1+O\left(2^{-\log ^{2} x}\right)\right) \exp \{\mu(\mathscr{B})\} .
$$

Поэтому везде в дальнейшем считаем, что $\alpha^{1}(\mathscr{B}) \geqslant \varkappa$, то есть достаточно велико.

Положим для краткости

$$
\mathscr{B}_{[2,3]}^{(2)}=\mathscr{B}_{[2]}^{(2)} \cup \mathscr{B}_{[3]}^{(2)} \text {, }
$$

а также

$$
\mu_{3}(\mathscr{B})=\alpha^{1}(\mathscr{B})-\frac{1}{2} \alpha^{2}(\mathscr{B})+\frac{1}{3} \alpha^{3}(\mathscr{B})-\alpha^{1}\left(\mathscr{B}_{[2,3]}^{(2)}\right) .
$$

Следствие 3. Пусть $I=(X, f)$ является $(3, \varkappa, q, c)$-ординарной функциональной парой, $\mathscr{B} \subseteq \mathbb{A}_{\hat{3}}(I)$, Тогда при достаточно больших $\varkappa$

$$
\tilde{S}(\mathscr{B})=\left(1+O\left(2^{-\log ^{2} x}\right)\right) \exp \left\{\mu_{3}(\mathscr{B})\right\} .
$$

Доказательство. Положим $j_{0}=1 /(3 c r)$. В силу (22) и (23) с $r=2$ и $\Delta=3$ при достаточно больших $\varkappa$

$$
\begin{aligned}
\alpha^{1}\left(\mathscr{B}^{(2)}\right)-\alpha^{1}\left(\mathscr{B}_{[2,3]}^{(2)}\right) & =\sum_{j \geqslant 4} \alpha^{1}\left(\mathscr{B}_{[j]}^{(2)}\right) \\
& \leqslant \sum_{4 \leqslant j \leqslant j_{0}} 2^{-(j-4) x-(3 / 2) \log ^{2} x}+\sum_{j>j_{0}} 2^{-j x / 2} \leqslant 2^{1-(3 / 2) \log ^{2} x} .
\end{aligned}
$$

Теперь (36) следует из (24) и (37).

Следствие 4. Пусть $I=(X, f)$ является $(3, x, q, c)$-ординарной функциональной парой, $r_{0}=\lceil\varkappa /(2 q)\rceil, \mathscr{B} \subseteq \mathscr{A}_{\hat{r}_{0}}(I), \widehat{\mathscr{B}}=\mathscr{B} \cap \mathscr{A}_{\hat{3}}(I)$. Тогда при достаточно больиих $\varkappa$

$$
\tilde{S}(\mathscr{B})=\left(1+O\left(2^{-\log ^{2} x}\right)\right) \exp \left\{\mu_{3}(\widehat{\not})\right\} .
$$

Утверждение вытекает из леммы 5 при $\Delta=3$ и из (36). 


\section{4. Ограниченные последовательности}

В предыдущем разделе была получена асимптотика сумм $\tilde{S}(\mathscr{B})$. В следующих разделах будет найдена оценка $\pi(B)$ и установлена связь между суммами $\tilde{S}(\mathscr{B})$ и $S(\mathscr{B})$.

В [3] было введено понятие $\alpha_{n}$-ограниченной последовательности. Если последовательность является ограниченной, то сумма последовательности приближается суммой типичных членов последовательности. В данном разделе доказывается, что последовательность $\tilde{S}_{k}(\mathscr{B})$ является $\alpha^{1}(\mathscr{B})$-ограниченной.

Последовательность $\left\{\sigma_{n, j}\right\}_{n, j=0}^{\infty}$ называется $\alpha_{n}$-ограниченной, если при любом $j$ выполняется неравенство

$$
(j+1) \sigma_{n, j+1} \leqslant \sigma_{n, j} \alpha_{n} .
$$

Для $\alpha_{n}$-ограниченных последовательностей справедливы следующие утверждения, доказанные в [3].

Теорема 6. Пусть последовательность $\left\{\sigma_{n, j}\right\}_{n, j=0}^{\infty}$ является $\alpha_{n}$-ограниченной,

$$
\begin{gathered}
\lim _{n \rightarrow \infty} \omega_{n}=\infty, \quad \theta_{n}=\alpha_{n}+\omega_{n} \sqrt{\alpha_{n}}, \quad \sigma_{n}=\sum_{j \geqslant 0} \sigma_{n, j}, \quad \alpha_{n, j}=(j+1) \sigma_{n, j+1} / \sigma_{n, j}, \\
\beta_{n}=\min _{j \leqslant \theta_{n}}\left\{\alpha_{n, j}\right\}, \quad \tau_{n}=\beta_{n}-\omega_{n} \sqrt{\beta_{n}}, \quad \tilde{\sigma}_{n}=\sum_{\tau_{n} \leqslant j \leqslant \theta_{n}} \sigma_{n, j} .
\end{gathered}
$$

Tогда при $n \rightarrow \infty$

$$
\tilde{\sigma}_{n}=\sigma_{n}\left(1-\varepsilon_{n}\right)
$$

где $\lim _{n \rightarrow \infty} \varepsilon_{n}=0$.

При этом $\varepsilon_{n}=O\left(e^{-\omega_{n}^{2} / 4}\right)$, если $\omega_{n} \leqslant \sqrt{\alpha_{n}}, u \varepsilon_{n}=O\left(\omega_{n}^{-1} e^{-\omega_{n}^{2} / 2}\right)$, если $\omega_{n}=o\left(\left(\alpha_{n}\right)^{1 / 6}\right)$.

Следствие 5. Пусть выполнены условия теоремы 6 и $\omega_{n}=o\left(\left(\alpha_{n}\right)^{1 / 6}\right)$. Тогда при $n \rightarrow \infty$

$$
\sigma_{n}=\tilde{\sigma}_{n}\left(1+O\left(\omega_{n}^{-1} e^{-\omega_{n}^{2} / 2}\right)\right)
$$

Пусть функционал $\pi: 2^{X} \rightarrow(0,1]$ удовлетворяет условию $(10)$. В дальнейшем для краткости полагаем

$$
\sigma=\tilde{S}(\mathscr{B})=\sum_{A \in C(\mathscr{B})} f(A) \pi(A), \quad \sigma_{k}=\tilde{S}_{k}(\mathscr{B})=\sum_{A \in C_{k}(\mathscr{B})} f(A) \pi(A) .
$$

Лемма 6. Для любой функциональной пары $I=(X, f)$ и любого челого числа $k \geqslant 0$

$$
(k+1) \sum_{A \in C_{k+1}(\mathscr{B})} f(A) \pi(A)=\sum_{B \in C_{k}(\mathscr{B})} f(B) \sum_{D \in \mathscr{B}_{\bar{B}}} f(D) \pi(B \cup D) .
$$

Доказательство. Правая часть (38) равна сумме произведений $f(B) f(D) \pi(B \cup D)$ по всем упорядоченным парам $(B, D)$ таким, что $B \in C_{k}(\mathscr{B}), D \in \mathscr{B}_{\bar{B}}$. Для каждой такой пары множество $A=B \cup D$ принадлежит семейству $C_{k+1}(\mathscr{B})$. При этом

$$
f(A) \pi(A)=f(B \cup D) \pi(B \cup D)=f(B) f(D) \pi(B \cup D) .
$$

Таким образом, каждая пара $(B, D)$ определяет множество $A \in C_{k+1}(\mathscr{B})$, причем каждое множество $A$ порождается $k+1$ раз. Отсюда следует (38). 
Пусть $I=(X, f)-$ функщиональная пара, $A \subseteq X, \mathcal{N} \subseteq 2^{X}$. Через $\eta_{\mathcal{N}}(A)$ обозначим число компонент множества $A$, принадлежащих семейству $\mathcal{N}$. Если $k$ - натуральное число, $\mathscr{B} \subseteq \mathscr{A}(I)$, то положим

$$
C_{k}(\mathscr{B} \mid \mathcal{N})=\left\{A \in C(\mathscr{B}): \eta_{\mathcal{N}}(A)=k\right\}
$$

Для натуральных $\tau \leqslant \theta$ положим для краткости

$$
C_{[\tau, \theta]}(\mathscr{B} \mid N)=\bigcup_{\tau \leqslant j \leqslant \theta} C_{j}(\mathscr{B} \mid N) .
$$

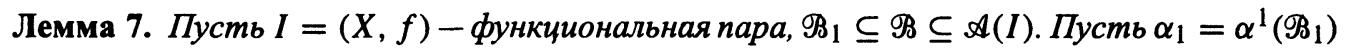
достаточно велико. Пусть также

$$
\theta_{1}=\alpha_{1}+\alpha_{1}^{9 / 14}, \quad \beta_{1}=\min _{j \leqslant \theta_{1}}\left\{(j+1) \tilde{S}_{j+1}\left(\mathscr{B}_{1}\right) / \tilde{S}_{j}\left(\mathscr{B}_{1}\right)\right\}, \quad \tau_{1}=\beta_{1}-\alpha_{1}^{1 / 7} \sqrt{\beta_{1}} .
$$

Тогда

$$
\tilde{S}(\mathscr{B})=\left(1+O\left(\alpha_{1}^{-1 / 7} e^{-\alpha_{1}^{2 / 7} / 2}\right)\right) \sum_{B \in C_{\left[\tau_{1}, \theta_{1}\right]}\left(\mathscr{B} \mid \mathscr{B}_{1}\right)} f(B) \pi(B) .
$$

Доказательство. Для доказательства утверждения воспользуемся следствием 5. Поло-

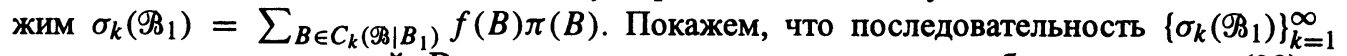
является $\alpha_{1}$-ограниченной. В самом деле, аналогично тому, как было получено (38), с использованием (10) получаем, что

$$
\begin{aligned}
(k+1) \sum_{B \in C_{k+1}\left(\mathscr{B} \mid B_{1}\right)} f(B) \pi(B) & =\sum_{B \in C_{k}\left(\mathscr{B} \mid B_{1}\right)} f(B) \sum_{C \in \mathscr{B}_{1 \bar{B}}} f(C) \pi(B \cup C) \\
& \leqslant \sum_{B \in C_{k}\left(\mathscr{B} \mid B_{1}\right)} f(B) \pi(B) \sum_{C \in \mathscr{B}_{1 \bar{B}}} f(C) \\
& \leqslant \alpha^{1}\left(\mathscr{B}_{1}\right) \sum_{B \in C_{k}\left(\mathscr{B} \mid B_{1}\right)} f(B) \pi(B) .
\end{aligned}
$$

Отсюда следует (39).

Теорема 7. Пусть $I=(X, f)-$ функциональная пара. Пусть семейства $\mathscr{B}_{1} \subseteq \mathscr{B} \subseteq \mathscr{A}(I)$ и $\mathscr{B}_{2}=\mathscr{B} \backslash \mathscr{B}_{1}$ таковы, что $\alpha_{1}=\alpha^{1}\left(\mathscr{B}_{1}\right)$ u $\alpha_{2}=\alpha^{1}\left(\mathscr{B}_{2}\right)$ достаточно велики. Пусть также

$$
\begin{array}{lll}
\theta_{1}=\alpha_{1}+\alpha_{1}^{9 / 14}, & \beta_{1}=\min _{j \leqslant \theta_{1}}\left\{(j+1) \tilde{S}_{j+1}\left(\mathscr{B}_{1}\right) / \tilde{S}_{j}\left(\mathscr{B}_{1}\right)\right\}, & \tau_{1}=\beta_{1}-\alpha_{1}^{1 / 7} \sqrt{\beta_{1}}, \\
\theta_{2}=\alpha_{2}+\alpha_{2}^{9 / 14}, & \beta_{2}=\min _{j \leqslant \theta_{2}}\left\{(j+1) \tilde{S}_{j+1}\left(\mathscr{B}_{2}\right) / \tilde{S}_{j}\left(\mathscr{B}_{2}\right)\right\}, & \tau_{2}=\beta_{2}-\alpha_{2}^{1 / 7} \sqrt{\beta_{2}} .
\end{array}
$$

Тогда

$$
\tilde{S}(\mathscr{B})=\left(1+O\left(\alpha_{1}^{-1 / 7} e^{-\alpha_{1}^{2 / 7} / 2}+\alpha_{2}^{-1 / 7} e^{-\alpha_{2}^{2 / 7} / 2}\right)\right) \sum_{B \in C_{\left[\tau_{1}, \theta_{1}\right]}\left(\mathscr{B} \mid \mathscr{B}_{1}\right) \cap C_{\left[\tau_{2}, \theta_{2}\right]}\left(\mathscr{B} \mid \mathscr{B}_{2}\right)} f(B) \pi(B) .
$$


Доказательство. Для доказательства утверждения воспользуемся следствием 5. Заметим, что

$$
\sum_{B \in C_{\left[\tau_{1}, \theta_{1}\right]}\left(\mathscr{B} \mid B_{1}\right)} f(B)=\sum_{A \in C\left(\mathscr{B}_{2}\right)} f(A) \sum_{C \in C_{\left[\tau_{1}, \theta_{1}\right]}\left(\mathscr{B}_{1 \bar{A}}\right)} f(C) \pi(A \cup C) .
$$

Положим

$$
\sigma_{k}\left(\mathscr{B}_{2}\right)=\sum_{A \in C_{k}\left(\mathscr{B}_{2}\right)} f(A) \sum_{C \in C_{\left[\tau_{1}, \theta_{1}\right]}\left(\mathscr{B}_{1 \bar{A}}\right)} f(C) \pi(A \cup C) .
$$

Покажем, что последовательность $\left\{\sigma_{k}\left(\mathscr{B}_{2}\right)\right\}_{k=1}^{\infty}$ является $\alpha_{2}$-ограниченной. Аналогично тому, как было получено (38), с использованием (10) находим, что

$$
\begin{aligned}
(k+1) \sum_{A \in C_{k+1}\left(\mathscr{B}_{2}\right)} & f(A) \sum_{C \in C_{\left[\tau_{1}, \theta_{1}\right]\left(\mathscr{B}_{1} \bar{A}\right)} f(C) \pi(A \cup C)} f(A) \sum_{B \in \mathscr{B}_{2} \bar{A}} f(B) \sum_{C \in C_{\left.\left[\tau_{1}, \theta_{1}\right] \mathscr{P}_{1} \overline{A \cup B}\right)} f(C) \pi(A \cup B \cup C)} f(C) \pi(A \cup C) \\
& \leqslant \sum_{A \in C_{k}\left(\mathscr{B}_{2}\right)} f(A) \sum_{B \in \mathscr{B}_{k}\left(\mathscr{B}_{2}\right)} f(B) \sum_{C \in C_{\left[\tau_{1}, \theta_{1}\right]}\left(\mathscr{B}_{1 \bar{A}}\right)} f(C) \sum_{C \in C_{\left[\tau_{1}, \theta_{1}\right]}\left(\mathscr{B}_{1} \overline{\bar{A}}\right)} f(C) \pi(A \cup C) .
\end{aligned}
$$

Отсюда и из (39) следует (40).

\section{5. Оценки $\tilde{S}(\mathscr{B})$ для $d$-связных множеств}

В данном разделе получена оценка $\pi(B)$ для множеств, $d$-компоненты которых имеют мощность, большую 1 (лемма 12 ). Это позволит при оценке $\pi(B)$ ограничиться рассмотрением только тех множеств, компоненты которых имеют мощность 1 и находятся на достаточно большом расстоянии друг от друга.

Пусть $\rho_{G}(u, v)$ - расстояние в графе $G$ между вершинами $u$ и $v$. Последовательность $\left(w_{0}, w_{1}, \ldots, w_{k}\right)$ называется $d$-цепью длины $k$, соединяющей $u$ и $v$, если $u=w_{0}, v=w_{k}$ и $\rho_{G}\left(w_{i-1}, w_{i}\right) \leqslant d$ при $i=1,2, \ldots, k$. Подмножество $A \subseteq X$ называется $d$-связным, если для любых $u, v$ из $A$ существует $d$-цепь, состоящая из элементов множества $A$ и соединяющая $u$ и $v$.

Пусть $I=(X, f)-$ функциональная пара. Множество всех $d$-связных подмножеств вершин графа $G_{I}$ обозначается через $\mathscr{A}^{d}(I)$. Положим

$$
\mathscr{A}_{k}^{d}(I)=\left\{A \in \mathbb{A}^{d}(I):|A|=k\right\}, \quad \mathscr{A}_{\hat{k}}^{d}(I)=\bigcup_{i \leqslant k} \mathscr{A}_{i}^{d}(I) .
$$

Подмножество $B \subseteq A$ называется $d$-компонентой множества $A$ (используется обозначение $B \vdash_{d} A$ ), если $B \in \mathscr{A}^{d}(I)$, но для любого $D \in \mathscr{A}^{d}(I)$ неверно, что $B \subset P \subseteq A$. Если $\mathscr{D} \subseteq A^{d}(I)$, то положим

$$
\begin{gathered}
C^{d}(\mathscr{D})=\left\{A \subseteq X: B \vdash_{d} A \Rightarrow B \in \mathscr{D}\right\}, \\
\alpha^{1, d}(\mathscr{D})=\sum_{B \in \mathscr{D}} f(B), \quad \tilde{S}^{d}(\mathscr{D})=\sum_{B \in C^{d}(\mathscr{D})} f(B) \pi(B) .
\end{gathered}
$$


Через $\eta^{d}(A)$ обозначается число $d$-компонент множества $A$. Для $\mathscr{D} \subseteq A^{d}(I)$ положим

$$
C_{k}^{d}(\mathscr{D})=\left\{A \in C^{d}(\mathscr{D}): \eta^{d}(A)=k\right\} .
$$

Для $\mathscr{B} \subseteq A(I)$ положим

$$
\begin{aligned}
& \mathscr{B}^{\langle d, 1\rangle}=C(\mathscr{B}) \cap \mathscr{A}^{d}(I), \\
& \mathscr{B}_{\langle-, \Delta\rangle}^{\langle d, 1\rangle}=\left\{A \in \mathscr{B}^{\langle d, 1\rangle}: B \vdash A \Rightarrow B \in \mathscr{B},|B|=\Delta\right\}, \\
& \mathscr{B}_{\langle-, \hat{\Delta}\rangle}^{\langle d, 1\rangle}=\left\{A \in \mathscr{B}^{\langle d, 1\rangle}: B \vdash A \Rightarrow B \in \mathscr{B},|B| \leqslant \Delta\right\}, \\
& \mathscr{B}_{\langle k,-\rangle}^{\langle d, 1\rangle}=\left\{A \in \mathscr{B}^{\langle d, 1\rangle}:|A|=k\right\}, \\
& \mathscr{B}_{\langle\hat{k},-\rangle}^{\langle d, 1\rangle}=\bigcup_{j \leqslant k} \mathscr{B}_{\langle j,-\rangle}^{\langle d, 1\rangle}, \\
& \mathscr{B}_{\langle\bar{k},-\rangle}^{\langle d, 1\rangle}=\mathscr{B}^{\langle d, 1\rangle} \backslash \mathscr{B}_{\langle\hat{k},-\rangle}^{\langle d, 1\rangle}, \\
& \mathscr{B}_{\langle k, \Delta\rangle}^{\langle d, 1\rangle}=\mathscr{B}_{\langle-, \Delta\rangle}^{\langle d, 1\rangle} \cap \mathscr{B}_{\langle k,-\rangle}^{\langle d, 1\rangle} .
\end{aligned}
$$

Для $v \in X$ положим

$$
\mathscr{A}_{\{v\}, j}^{d}(I)=\left\{A \in \mathscr{A}^{d}(I):|A|=j, A \#\{v\}\right\} .
$$

Лемма 8. Пусть функциональная пара $I=(X, f)$ такова, что для всякого $v \in X$, всякого натурального т и некоторого натурального $c$

$$
\left|A_{\{v\}, \hat{m}}(I)\right| \leqslant \varkappa^{c m}
$$

Тогда для всех натуральных $d u j$

$$
\left|\mathscr{A}_{\{v\}, j}^{d}(I)\right| \leqslant \varkappa^{c d j}
$$

Доказательство. Заметим, что $B \in \mathscr{A}_{\{v\}, j}^{d}(I)$ означает, что существует вершина $u \in B$ такая, что $u \# v$, то есть $\rho_{G_{I}}(u, v)=1$, и что множество $B$ является $d$-связным. Для выбора множества $B \in \mathscr{A}_{\{v\}, j}^{d}(I)$ достаточно указать вершину $u \in X$ такую, что $\rho_{G_{I}}(u, v)=1$, и $d$-связное множество $B$ такое, что $u \in B$.

Пусть $n_{d}(\{u\}, a)$ - число всех $d$-связных множеств $A \subseteq X$ таких, что $|A|=a$ и $u \in A$. Каждая вершина $u \in X$ соединена в графе $G_{I}$ с вершинами из множества $\mathscr{A}_{\{u\}, 1}$, а по условию число таких вершин не превышает $\varkappa^{c}$. Таким образом, граф $G_{I}$ удовлетворяет условиям леммы 6.1 из [4] с $\sigma=\varkappa^{c}$, и следовательно,

$$
n_{d}(\{u\}, a) \leqslant\left(4 \varkappa^{c d}\right)^{a-1} .
$$

Для заданной вершины $v \in X$ вершину $u \in X$ такую, что $\rho_{G_{I}}(u, v)$, можно выбрать не более, чем $\varkappa^{c}$ способами, следовательно, при достаточно больших $\varkappa$

$$
\left|\mathbb{A}_{\{v\}, j}^{d}(I)\right| \leqslant\left(4 \varkappa^{c d}\right)^{j-1} \varkappa^{c} \leqslant \varkappa^{c d j} .
$$

Лемма доказана.

Лемма 9. Пусть $I=(X, f)$ является $(\Delta, \varkappa, q, c)$-ординарной функциональной парой, $d$ - натуральное число, $r_{0}=\lceil\varkappa /(2 q)\rceil, \mathscr{B} \subseteq A_{\hat{r}_{0}}(I)$. Тогда

$$
\alpha^{1, d}\left(\mathscr{B} \frac{\langle\bar{\Delta},-\rangle\rangle}{\langle d\rangle}\right) \leqslant 2^{-\log ^{2} x} .
$$


Доказательство. При $j \geqslant \Delta+1$ из леммы 8 и (20) следует, что

$$
\begin{aligned}
\alpha^{1, d}\left(\mathscr{B}_{(j,-)}^{\langle d, 1\rangle}\right) & =\alpha^{1, d}\left(\mathscr{B}_{\left\langle j, \hat{r}_{0}\right)}^{\langle d, 1\rangle}\right)=\sum_{B \in C(\mathscr{B}) \cap A_{j}^{d}(I)} f(B) \\
& \leqslant \sum_{v \in X} \sum_{B \in C(\mathscr{B}) \cap \mathbb{A}_{\{v\}, j}^{d}(I)} f(B) \leqslant \sum_{v \in X}\left|\mathbb{A}_{\langle v\}, j}^{d}(I)\right| 2^{-j\left(x-q\left(r_{0}-1\right) / 2\right)} \\
& \leqslant|X| \varkappa^{c d j} 2^{-j \varkappa+q j r_{0} / 2} \leqslant 2^{-\varkappa(j-\Delta-1)-2 \log ^{2} \varkappa_{\chi^{c d j}} 2^{q j r_{0} / 2}}
\end{aligned}
$$

Положим $j_{0}=1 /(3 c d)$. Аналогично тому, как были получены (22), (23) (см. следствие 5.6 в работе [4]), находим, что

$$
\begin{aligned}
\alpha^{1, d}\left(\mathscr{B}_{(\bar{\Delta},-\rangle}^{\langle d, 1\rangle}\right) & =\sum_{\Delta+1 \leqslant j} \alpha^{1, d}\left(\mathscr{B}_{\langle j,-\rangle}^{\langle d, 1\rangle}\right) \\
& \leqslant \sum_{\Delta+1 \leqslant j \leqslant j_{0}} 2^{-\varkappa(j-\Delta-1)-(3 / 2) \log ^{2} \varkappa}+\sum_{j_{0} \leqslant j} 2^{-\varkappa j / 2} \leqslant 2^{-\log ^{2} \varkappa} .
\end{aligned}
$$

Лемма доказана.

Лемма 10. Пусть $I=(X, f)$ является $(\Delta, x, q, c)$-ординарной функциональной парой, $r_{0}=\lceil\varkappa /(2 q)\rceil, \mathscr{B} \subseteq A_{\hat{r}_{0}}(I), d-$-натуральное число. Тогда

$$
\tilde{S}(\mathscr{B})=\left(1+O\left(2^{-\log ^{2} \varkappa}\right)\right) \tilde{S}^{d}\left(\mathscr{B}_{\langle\hat{\Delta}, \hat{\Delta}\rangle}^{\langle d, 1\rangle}\right) .
$$

Доказательство. Поскольку

$$
C(\mathscr{B})=C^{d}\left(C(\mathscr{B}) \cap \mathscr{A}^{d}(I)\right)=C^{d}\left(\mathscr{B}^{\langle d, 1\rangle}\right),
$$

To

$$
\tilde{S}(\mathscr{B})=\tilde{S}^{d}\left(\mathscr{B}^{\langle d, 1\rangle}\right) .
$$

Аналогично тому, как это сделано при доказательстве леммы 5, из (41) получаем, что

$$
\begin{aligned}
\tilde{S}^{d}\left(\mathscr{B}_{\langle\hat{\Delta}, \hat{\Delta}\rangle}^{\langle d, 1\rangle}\right) & \left.\leqslant \tilde{S}(\mathscr{B})=\tilde{S}^{d}\left(\mathscr{B}^{\langle d, 1\rangle}\right)=\tilde{S}_{(\mathscr{B}}^{d}{ }_{\langle\hat{\Delta}, \hat{\Delta}\rangle}^{\langle d, 1\rangle} \cup \mathscr{B}_{\langle\bar{\Delta},-\rangle}^{\langle d, 1\rangle}\right) \\
& \leqslant \tilde{S}^{d}\left(\mathscr{B}_{\langle\hat{\Delta}, \hat{\Delta})}^{\langle d, 1\rangle}\right) \exp \left\{\alpha^{1, d}\left(\mathscr{B}_{\langle\bar{\Delta},-\rangle}^{\langle d, 1\rangle}\right)\right\} \leqslant\left(1+O\left(2^{-\log ^{2} x}\right)\right) \tilde{S}^{d}\left(\mathscr{B}_{\langle\hat{\Delta}, \hat{\Delta}\rangle}^{\langle d, 1\rangle}\right) .
\end{aligned}
$$

Лемма доказана.

Для $\mathscr{D} \subseteq A^{d}(I)$, целого $j$ и $B \subseteq \mathscr{D}$ положим

$$
\begin{gathered}
\sigma_{j}^{d}=\tilde{S}_{j}^{d}(\mathscr{D})=\sum_{B \in C_{j}^{d}(\mathscr{D})} f(B) \pi(B), \\
\mathscr{D}_{[j]}=\{B \in \mathscr{D}:|B|=j\}, \quad \mathscr{D}_{B}=\left\{C \in \mathscr{D}:(B \cup C) \in \mathscr{A}^{d}(I)\right\} .
\end{gathered}
$$

Лемма 11. Пусть $I=(X, f)$ является $(-, \varkappa,-, c)$-ординарной функциональной парой, $t, d$ - натуральные числа,

$\mathscr{B} \subseteq \mathscr{A}(I), \quad \mathscr{D} \subseteq \mathscr{B}_{\langle\hat{i},-\rangle}^{\langle d, 1\rangle}, \quad \alpha=\alpha^{1, d}(\mathscr{D}), \quad \theta=\alpha+\alpha^{9 / 14}, \quad \beta=\min _{j \leqslant \theta}\left\{(j+1) \sigma_{j+1}^{d} / \sigma_{j}^{d}\right\}$,

Тогда существует положительное число $\varepsilon_{1}$ такое, что

$$
\beta \geqslant \alpha\left(1-\varepsilon_{1} 2^{-\varkappa} \varkappa^{c d}\right)
$$


Доказательство. Оценим $(j+1) \sigma_{j+1}^{d} / \sigma_{j}^{d}$ при $j \leqslant \theta$. Аналогично тому, как было получено (38), из (10) находим, что

$$
\begin{aligned}
(j+1) \sum_{B \in C_{j+1}^{d}(\mathscr{D})} f(B) \pi(B) & =\sum_{B \in C_{j}^{d}(\mathscr{D})} f(B) \sum_{D \in \mathscr{D}_{\bar{B}}} f(D) \pi(B \cup D) \\
& \geqslant \sum_{B \in C_{j}^{d}(\mathscr{D})} f(B) \pi(B) \sum_{D \in \mathscr{D}_{\bar{B}}} f(D) \pi(D) .
\end{aligned}
$$

Заметим, что если $D \in \mathscr{D}_{\bar{B}} \subseteq \mathscr{B}_{\langle\hat{t},-\rangle}^{\langle d, 1\rangle}$, то $|D| \leqslant t$. Воспользуемся (21) с $m=t, r=2$ и $v=1:$

$$
\alpha^{1}\left(\mathscr{B}_{D}^{(2)}\right)=\sum_{j \geqslant 2} \alpha^{1}\left(\mathscr{B}_{D,[j]}^{(2)}\right) \leqslant|D| \sum_{j \geqslant 2} 2^{-j \varkappa+j q t / 2+3 j} \varkappa^{2 j c} \leqslant 2 t 2^{-2 \varkappa+q t+6} \varkappa^{4 c} .
$$

Следовательно,

$$
\pi(D) \geqslant \exp \left\{-t 2^{-2 x+q t+7} \varkappa^{4 c}\right\}
$$

Тогда

$$
\begin{aligned}
& \sum_{B \in C_{j}^{d}(\mathscr{D})} f(B) \pi(B) \sum_{D \in \mathscr{D}_{\bar{B}}} f(D) \pi(D)=\left(1+O\left(2^{-2 \varkappa} \varkappa^{4 c}\right)\right) \sum_{B \in C_{j}^{d}(\mathscr{D})} f(B) \pi(B) \alpha^{1, d}\left(\mathscr{D}_{\bar{B}}\right) \\
& =\left(\sum_{B \in C_{j}^{d}(\mathscr{D})} f(B) \pi(B) \alpha^{1, d}(\mathscr{D})-\sum_{B \in C_{j}^{d}(\mathscr{D})} f(B) \pi(B) \alpha^{1, d}\left(\mathscr{D}_{B}\right)\right)\left(1+O\left(2^{-2 \varkappa} \varkappa^{4 c}\right)\right)
\end{aligned}
$$

Оценим $\alpha^{1, d}\left(\mathscr{D}_{B}\right)$ при $B \in C_{j}^{d}(\mathscr{D})$. Множество $B$ состоит не более, чем из $\theta d$-компонент, каждая компонента имеет мощность, не превосходящую $t$. Поскольку $\alpha>1$, то $\theta \leqslant 2 \alpha$, и следовательно, $|B| \leqslant t \theta \leqslant 2 t \alpha^{1, d}(\mathscr{D})$. Аналогично (21) с $m=t, r=1$ и $v=1$

$$
\begin{aligned}
\alpha^{1, d}\left(\mathscr{D}_{B}\right) & =\sum_{j \geqslant 1} \alpha^{1, d}\left(\mathscr{D}_{B,[j]}\right) \leqslant|B| \sum_{j \geqslant 1} \varkappa^{c d j} 2^{-j \varkappa+j q t / 2} \\
& \leqslant 2|B| 2^{-\varkappa+q t / 2} \varkappa^{c d} \leqslant 4 t 2^{-\varkappa+q t / 2} \varkappa^{c d} \alpha^{1, d}(\mathscr{D}) .
\end{aligned}
$$

При $j \leqslant \theta$ для некоторого $\varepsilon_{1}>0$ из (42)-(44) получаем, что

$$
\begin{aligned}
\frac{(j+1) \sigma_{j+1}}{\sigma_{j}} \geqslant & \left(\alpha^{1, d}(\mathscr{D})-\sum_{B \in C_{j}^{d}(\mathscr{D})} f(B) \pi(B) \alpha^{1, d}\left(\mathscr{D}_{B}\right) / \sum_{B \in C_{j}^{d}(\mathscr{D})} f(B) \pi(B)\right) \\
& \times\left(1+O\left(2^{-2 \varkappa} \varkappa^{4 c}\right)\right) \\
\geqslant & \alpha^{1, d}(\mathscr{D})\left(1-\varepsilon_{1} 2^{-\chi} \varkappa^{c d}\right) .
\end{aligned}
$$

Лемма доказана.

Следствие 6. Пусть $I=(X, f)$ является $(-, \varkappa,-, c)$-ординарной функциональной парой, $t, d$ - натуральные числа,

$\mathscr{B} \subseteq \mathscr{A}(I), \quad \mathscr{D} \subseteq \mathscr{B}_{\langle\hat{t},-\rangle}^{\langle d, 1\rangle}, \quad \alpha=\alpha^{1, d}(\mathscr{D}), \quad \theta=\alpha+\alpha^{9 / 14}, \quad \beta=\min _{j \leqslant \theta}\left\{(j+1) \sigma_{j+1}^{d} / \sigma_{j}^{d}\right\}$. 
Пусть $\tau=\beta-\alpha^{1 / 7} \sqrt{\beta}$, тогда существует положстельное число $\varepsilon_{2}$ такое, что

$$
\left(\alpha-\alpha^{9 / 14}\right)\left(1-\varepsilon_{2} 2^{-\varkappa} \varkappa^{c d}\right) \leqslant \tau \leqslant\left(\alpha-\alpha^{9 / 14}\right) .
$$

Доказательство. Заметим, что функщия $x-\alpha^{1 / 7} \sqrt{x}$ возрастает при $x \geqslant \alpha^{2 / 7}$ и $\alpha \geqslant 1$. В силу леммы 11 найдется $\varepsilon_{1}>0$ такое, что

$$
\tau=\beta-\alpha^{1 / 7} \geqslant \alpha\left(1-\varepsilon_{1} 2^{-x} \varkappa^{c d}\right)-\alpha^{9 / 4} \sqrt{1-\varepsilon_{1} 2^{-\chi} \varkappa^{c d}} .
$$

С использованием неравенства $\sqrt{1-y} \leqslant 1-y / 2$ нетрудно проверить, что при $\alpha \geqslant 1$

$$
\alpha^{9 / 4} \sqrt{1-\varepsilon_{1} 2^{-\varkappa} \varkappa^{c d}} \geqslant\left(\alpha-\alpha^{9 / 14}\right)\left(1-\varepsilon_{2} 2^{-\varkappa} \varkappa^{c d}\right),
$$

где $\varepsilon_{2}=3 \varepsilon_{1} / 2$. Отсюда и из (45) следует утверждение.

Лемма 12. Пусть $I=(X, f)$ является $(3, x, q, c)$-ординарной функциональной парой, $\mathscr{B} \subseteq \mathscr{A}_{\hat{3}}(I), \mathscr{D} \subseteq \mathscr{B}_{\langle\hat{3}, \hat{3}\rangle}^{\langle d, 1\rangle} \backslash \mathscr{B}_{\langle 1,1\rangle}^{\langle d, 1\rangle}$. Пусть $\alpha=\alpha^{1, d}(\mathscr{D}), \theta=\alpha+\alpha^{9 / 14}$. Пусть также $B \in C_{[1, \theta]}^{d}(D)$. Тогда при достаточно больиих $\varkappa$

$$
1-2^{-(1 / 2) \log ^{2} x} \leqslant \pi(B)=\exp \left\{-\alpha\left(\mathscr{\Re}_{B}^{(2)}\right)\right\} \leqslant 1 .
$$

Доказательство. Второе неравенство очевидно. Докажем первое неравенство. Из включения $B \in C_{[1, \theta]}^{d}(D)$ следует, что $B$ состоит не более, чем из $\theta d$-компонент, и каждая компонента содержит не более трех элементов. Поскольку $\alpha>1$, то $\theta \leqslant 2 \alpha$, следовательно, $|B| \leqslant 3 \theta \leqslant 6 \alpha$.

Воспользуемся (21) с $m=3, r=2, v=1$

$$
\begin{aligned}
\alpha^{1}\left(\mathscr{B}_{B}^{(2)}\right) & =\sum_{j \geqslant 2} \alpha^{1}\left(\mathscr{B}_{B,[j]}^{(2)}\right) \leqslant|B| \sum_{j \geqslant 2} 2^{-j \varkappa+j q+3 j} \varkappa^{2 c j} \\
& \leqslant 2|B| 2^{-2 \varkappa+2 q+6} \varkappa^{4 c} \leqslant 3 \alpha^{1, d}(\mathscr{D}) 2^{-2 \varkappa+2 q+8} \varkappa^{4 c} .
\end{aligned}
$$

Оценим $\alpha^{1, d}(\mathscr{D})$. Из определения семейства $\mathscr{D}$ следует, что оно состоит из множеств, мощность которых не меньше 2 и не больше 3. Справедлива оценка

$$
\alpha^{1, d}(\mathscr{D})=\sum_{A \in \mathscr{D}} f(A) \leqslant \sum_{A \in \mathscr{A}_{2}^{d}(I)} f(A)+\sum_{A \in \mathscr{A}_{3}^{d}(I)} f(A) .
$$

Отсюда, аналогично (29) получаем, что

$$
\alpha^{1, d}(\mathscr{D}) \leqslant \varkappa^{c d} 2^{2 \varkappa-\log ^{2} x+q}+\varkappa^{2 c d} 2^{\varkappa-\log ^{2} x+2 q} \leqslant 2 \cdot 2^{2 \varkappa-\log ^{2} \varkappa} \varkappa^{c d} .
$$

Из (46) и (47) вытекает, что $\alpha^{1}\left(\mathscr{B}_{B}^{(2)}\right) \leqslant 6 \cdot 2^{2 x-\log ^{2} x} 2^{-2 x+2 q+8} \chi^{4 c d} \leqslant 2^{-(1 / 2) \log ^{2} x}$. Отсюда следует утверждение леммы.

Пусть $I=(X, f)-$ функциональная пара, $A \subseteq X, \mu \subseteq 2^{X}$. Через $\eta_{\mu}^{d}(A)$ обозначим число $d$-компонент множества $A$, принадлежащих семейству $M$. Если $k$ - натуральное число, $\mathscr{D} \subseteq \mathscr{A}^{d}(I)$, то положим

$$
C_{k}^{d}(\mathscr{D} \mid \mathcal{M})=\left\{A \in C(\mathscr{D}): \eta_{\mathcal{M}}^{d}(A)=k\right\} .
$$

Для натуральных $\tau \leqslant \theta$ положим для краткости

$$
C_{[\tau, \theta]}^{d}(\mathscr{D} \mid \mu)=\bigcup_{\tau \leqslant j \leqslant \theta} C_{j}^{d}(\mathscr{D} \mid \mu) .
$$


Теорема 8. Пусть $I=(X, f)$ является $(3, \varkappa, q, c)$-ординарной функциональной парой, $\mathscr{B} \subseteq \mathscr{A}_{\hat{3}}(I), \mathscr{D}=\mathscr{B}_{\langle\hat{3}, \hat{3}\rangle}^{\langle d, \hat{1}\rangle}, \mathscr{D}_{1}=\mathscr{B}_{\langle 1,1\rangle}^{\langle d, 1\rangle}, \mathscr{D}_{2}=\mathscr{D}_{\backslash} \mathscr{D}_{1} . \Pi$ ycmь $B \in C(\mathscr{B}), B=B_{1} \cup B_{2}$, гдe $B_{1} \in C^{d}\left(\mathscr{D}_{1}\right), B_{2} \in C^{d}(\mathscr{D}) \backslash C^{d}\left(\mathscr{D}_{1}\right) . \Pi y c m b$

$$
\begin{array}{lll}
\alpha_{1}=\alpha^{1, d}\left(D_{1}\right), & \theta_{1}=\alpha_{1}+\alpha_{1}^{9 / 14}, & \tau_{1}=\left(\alpha_{1}-\alpha_{1}^{9 / 14}\right)\left(1-\varepsilon_{1} 2^{-\varkappa} \varkappa^{c d}\right), \\
\alpha_{2}=\alpha^{1, d}\left(\mathscr{D}_{2}\right), & \theta_{2}=\alpha_{2}+\alpha_{2}^{9 / 14}, & \tau_{2}=\left(\alpha_{2}-\alpha_{2}^{9 / 14}\right)\left(1-\varepsilon_{2} 2^{-\varkappa} \varkappa^{c d}\right),
\end{array}
$$

где $\varepsilon_{1}, \varepsilon_{2}>0$. Пусть также

$$
\tilde{C}^{d}(\mathscr{B})=C_{\left[\tau_{1}, \theta_{1}\right]}^{d}\left(\mathscr{D} \mid \mathscr{D}_{1}\right) \cap C_{\left[\tau_{2}, \theta_{2}\right]}^{d}\left(\mathscr{D} \mid \mathscr{D}_{2}\right) .
$$

Тогда при достаточно больших $\varkappa$

$$
\tilde{S}(\mathscr{B})=\left(1+O\left(\alpha_{1}^{-1 / 7} e^{-\alpha_{1}^{2 / 7} / 2}+\alpha_{2}^{-1 / 7} e^{-\alpha_{2}^{2 / 7} / 2}\right)\right) \sum_{B \in \tilde{C}^{d}(\mathscr{B})} f(B) \pi\left(B_{1}\right) .
$$

Доказательство. Заметим, что при $\mathscr{B} \subseteq \mathscr{A}_{\hat{3}}(I)$

$$
C(\mathscr{B})=C^{d}\left(\mathscr{B}_{\langle-, \hat{3}\rangle}^{\langle d, 1\rangle}\right)
$$

Из (10) следует, что

$$
\begin{aligned}
\sum_{B \in C^{d}(\mathscr{D})} f(B) \pi\left(B_{1}\right) \geqslant \tilde{S}^{d}(\mathscr{D}) & =\sum_{B \in C^{d}(\mathscr{D})} f(B) \pi\left(B_{1} \cup B_{2}\right) \\
& \geqslant \sum_{B \in C^{d}(\mathscr{D})} f(B) \pi\left(B_{1}\right) \pi\left(B_{2}\right) \geqslant \sum_{B \in \tilde{C}^{d}(\mathscr{B})} f(B) \pi\left(B_{1}\right) \pi\left(B_{2}\right) .
\end{aligned}
$$

Теперь утверждение теоремы следует из теоремы 7 и лемм 10 и 12 .

\section{6. Оценки $S(\mathscr{B})$ для почти регулярных множеств}

В этом разделе будет установлена связь между суммами $\tilde{S}(\mathscr{B})$ и $S(\mathscr{B})$ для почти регулярных семейств связных множеств.

Пусть $I=(X, f)$ - функциональная пара, семейство $\mathscr{B} \subseteq A(I)$ назовем регулярным, если для любых $\{u\},\{v\} \in \mathscr{B}$ справедливо равенство

$$
\alpha^{1}\left(\mathscr{B}_{\{v\},[2]}^{(2)}\right)=\alpha^{1}\left(\mathscr{B}_{\{u\},[2\}}^{(2)}\right)
$$

Семейство $\mathscr{B} \subseteq A(I)$ назовем почти регулярным, если для любых $\{u\},\{v\} \in \mathscr{B}$ выполняется соотношение

$$
\alpha^{1}\left(\mathscr{B}_{\{v\},[2]}^{(2)}\right)=\left(1+O\left(2^{-\log ^{2} x}\right)\right) \alpha^{1}\left(\mathscr{B}_{\{u\},[2]}^{(2)}\right) .
$$

Для $\mathscr{B} \subseteq A(I)$ положим

$$
\mathscr{B}_{[3]}^{(1,2)}=\left\{\{\{v\}, l\{\{u\},\{w\}\}\}:\{v\} \in \mathscr{B},\{u, w\} \in \mathscr{B}_{\{v\}}\right\} .
$$


Лемма 13. Пусть $I=(X, f)$ является $(3, \chi, q, c)$-ординарной функииональной парой, $\mathscr{B} \subseteq \mathscr{A}_{\hat{1}}(I)$ является почти регулярным семейством. Пусть

$$
\alpha=\alpha^{1, d}\left(\mathscr{B}_{\langle 1,1\rangle}^{\langle d, 1\rangle}\right), \quad \theta=\alpha+\alpha^{9 / 14}, \quad \tau=\left(\alpha-\alpha^{9 / 14}\right)\left(1-\varepsilon 2^{-\varkappa} \varkappa^{c d}\right), \quad \varepsilon>0 .
$$

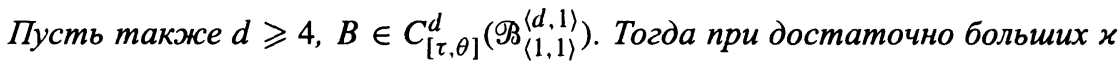

$$
\alpha^{1}\left(\mathscr{B}_{B}^{(2)}\right)=\left(1+O\left(2^{-\log ^{2} x}\right)\right) \alpha^{1}\left(\mathscr{B}_{[3]}^{(1,2)}\right) .
$$

Доказательство. Заметим, что в силу (16)

$$
\alpha^{1, d}\left(\mathscr{B}_{(1,1)}^{(d, 1)}\right)=\alpha^{1}(\mathscr{B})=\sum_{\{v\} \in \mathscr{B}} f(\{v\}) \leqslant|\mathscr{B}| 2^{-\varkappa} \leqslant|X| 2^{-\varkappa} \leqslant 2^{3 \varkappa-2 \log ^{2} \varkappa}
$$

Покажем, что при $B \in C_{[\tau, \theta]}^{d}\left(\mathscr{B}_{\langle 1,1\rangle}^{\langle d, 1\rangle}\right)$

$$
\alpha^{1}\left(\mathscr{B}_{B}^{(2)}\right)=\left(1+O\left(2^{-\log ^{2} x}\right)\right) \alpha^{1}\left(\mathscr{B}_{B,[2]}^{(2)}\right) .
$$

В силу (48) и (17)

$$
|B| \leqslant \theta \leqslant 2 \alpha=2 \cdot 2^{3 x-2 \log ^{2} x} .
$$

С использованием (16) и (21) при $m=1, r=2$ и $v=1$ получаем, что

$$
\begin{aligned}
\alpha^{1}\left(\mathscr{B}_{B}^{(2)}\right)-\alpha^{1}\left(\mathscr{R}_{B,[2]}^{(2)}\right) & =\sum_{j \geqslant 3} \alpha^{1}\left(\mathscr{B}_{B,[j]}^{(2)}\right) \leqslant|B| \sum_{j \geqslant 3} 2^{-j \varkappa+3 j} \varkappa^{2 c j} \\
& \leqslant 2 \cdot 2^{3 \varkappa-2 \log ^{2} \varkappa} \sum_{j \geqslant 3} 2^{-j \varkappa+3 j} \mathcal{\varkappa}^{2 c j} \leqslant 2^{-\log ^{2} \varkappa} .
\end{aligned}
$$

Отсюда следует (49). Далее, по формуле включения-исключения

$$
\begin{aligned}
\alpha^{1}\left(\mathscr{B}_{B,[2]}^{(2)}\right)=\alpha^{1}\left(\bigcup_{A \subseteq B} \mathscr{B}_{A,[2]}\right)= & \sum_{\{u\} \subseteq B} \alpha^{1}\left(\mathscr{B}_{\{u\},[2]}^{(2)}\right)-\sum_{\{u, v\} \subseteq B} \alpha^{1}\left(\mathscr{P}_{\{u\},[2]}^{(2)} \cap \mathscr{B}_{\{v\},[2]}^{(2)}\right) \\
& +\sum_{\{u, v, w\} \subseteq B} \alpha^{1}\left(\mathscr{P}_{\{u\},[2]}^{(2)} \cap \mathscr{B}_{\{v\},[2]}^{(2)} \cap \mathscr{B}_{\{w\},[2]}^{(2)}\right)-\ldots
\end{aligned}
$$

Пусть $H \in \mathscr{P}_{B,[2]}^{(2)}$, это значит, что существует вершина $v \in B$ такая, что $H \#\{v\}$. Если множество $B$ удовлетворяет условию леммы, то оно состоит из компонент (точек), расстояние между которыми не меньше $d$, тогда при $d \geqslant 4$ для любой вершины $u \in B$, отличной от $v$, справедливо соотношение $H \|\{u\}$. Следовательно, $\mathscr{B}_{\{v\},[2]}^{(2)} \cap \mathscr{B}_{\{u\},[2]}^{(2)}=\varnothing$, таким образом, все слагаемые, кроме первого, равняются нулю. Теперь из регулярности $\mathscr{B}$ для произвольного $\left\{v_{0}\right\} \in \mathscr{B}$ получаем, что

$$
\alpha^{1}\left(\mathscr{B}_{B,[2]}^{(2)}\right)=\sum_{\{v\} \subseteq B} \alpha^{1}\left(\mathscr{P}_{\{v\},[2]}^{(2)}\right)=\left(1+O\left(2^{-\log ^{2} x}\right)\right)|B| \alpha^{1}\left(\mathscr{B}_{\left\{v_{0}\right\},[2]}^{(2)}\right) .
$$

Поскольку $B \in C_{[\tau, \theta]}^{d}\left(\mathscr{B}_{(1,1\rangle}^{\langle d, 1\rangle}\right)$, в силу (48)

$$
|B|=\alpha^{1}(\mathscr{B})\left(1+O\left(\alpha^{-5 / 14}\right)\right)=\alpha^{1}(\mathscr{B})\left(1+O\left(2^{-15 x / 14}\right)\right) .
$$


Следовательно,

$$
\alpha^{1}\left(\mathscr{B}_{B,[2]}^{(2)}\right)=\left(1+O\left(2^{-\log ^{2} \varkappa}\right)\right) \alpha^{1}(\mathscr{B}) \alpha^{1}\left(\mathscr{B}_{\left\{v_{0}\right\},[2]}^{(2)}\right)
$$

Из (49), (51), определения семейства $\mathscr{B}_{[3]}^{(1,2)}$ и регулярности $\mathscr{B}$ следует, что

$$
\begin{aligned}
\alpha^{1}\left(\mathscr{B}_{B}^{(2)}\right) & =\left(1+O\left(2^{-\log ^{2} x}\right)\right) \alpha^{1}(\mathscr{B}) \alpha^{1}\left(\mathscr{B}_{\left\{v_{0}\right\},[2]}^{(2)}\right) \\
& =\left(1+O\left(2^{-\log ^{2} x}\right)\right) \sum_{\{v\} \in \mathscr{B}} f(\{v\}) \alpha^{1}\left(\mathscr{B}_{\{v\},[2]}^{(2)}\right) \\
& =\left(1+O\left(2^{-\log ^{2} x}\right) \sum_{\{v\} \in \mathscr{B}} f(\{v\}) \sum_{\{u, w\} \in \mathscr{B}_{\{v\}}} f(\{u\}) f(\{w\})\right. \\
& =\left(1+O\left(2^{-\log ^{2} x}\right)\right) \alpha^{1}\left(\mathscr{B}_{[3]}^{(1,2)}\right) .
\end{aligned}
$$

Лемма доказана.

Теорема 9. Пусть $I=(X, f)$ является $(3, \varkappa, q, c)$-ординарной функциональной парой, $\mathscr{B} \subseteq \mathscr{A}_{\hat{3}}(I)$ является почти регулярным семейством. Тогда

$$
S(\mathscr{B})=\left(1+O\left(2^{-(1 / 2) \log ^{2} x}\right)\right) \exp \left\{\hat{\mu}_{3}(\mathscr{B})\right\},
$$

где

$$
\hat{\mu}_{3}(\mathscr{B})=\alpha^{1}(\mathscr{B})-\frac{1}{2} \alpha^{2}(\mathscr{B})+\frac{1}{3} \alpha^{3}(\mathscr{B})-\alpha^{1}\left(\mathscr{B}_{[2,3]}^{(2)}\right)+\alpha^{1}\left(\mathscr{B}_{[3]}^{(1,2)}\right) .
$$

Утверждение вытекает из следствия 4, теоремы 8 и леммы 13.

Пусть $I=(X, f)$ - функциональная пара, семейства $\mathscr{B} \subseteq A(I)$ и $\mathscr{D} \subseteq A(I)$ назовем взаимно регулярными, если они являются почти регулярными, $\mathscr{B} \cap D=\varnothing$, а также для любых $\{u\},\{v\} \in \mathscr{B}$

$$
\alpha^{1}\left((\mathscr{B} \cup D)_{\{v\},[2]}^{(2)}\right)=\left(1+O\left(2^{-\log ^{2} x}\right)\right) \alpha^{1}\left((\mathscr{B} \cup D)_{\{u\},[2]}^{(2)}\right),
$$

а для любых $\{u\},\{v\} \in \mathscr{D}$

$$
\alpha^{1}\left((\mathscr{B} \cup D)_{\{v\},[2]}^{(2)}\right)=\left(1+O\left(2^{-\log ^{2} x}\right)\right) \alpha^{1}\left((\mathscr{B} \cup D)_{\{u\},[2]}^{(2)}\right) .
$$

Лемма 14. Пусть $I=(X, f)$ является $(3, \varkappa, q, c)$-ординарной функциональной парой, $\mathscr{B} \subseteq \mathscr{A}_{\hat{1}}(I)$ и $\mathscr{D} \subseteq \mathscr{A}_{\hat{\imath}}(I)$ являются взаимно регулярными семействами. Пусть

$$
\begin{aligned}
\alpha_{1} & =\alpha^{1, d}\left(\mathscr{P}_{\langle 1,1\rangle}^{\langle d, 1\rangle}\right), & \alpha_{2} & =\alpha^{1, d}\left(\mathscr{D}_{\langle 1,1\rangle}^{\langle d, 1\rangle}\right), \\
\theta_{1} & =\alpha_{1}+\alpha_{1}^{9 / 14}, & \theta_{2} & =\alpha_{2}+\alpha_{2}^{9 / 14}, \\
\tau_{1} & =\left(\alpha_{1}-\alpha_{1}^{9 / 14}\right)\left(1-\varepsilon_{1} 2^{-\varkappa} \varkappa^{c d}\right), & \tau_{2} & =\left(\alpha_{2}-\alpha_{2}^{9 / 14}\right)\left(1-\varepsilon_{2} 2^{-\varkappa} \varkappa^{c d}\right),
\end{aligned}
$$

$\varepsilon_{1}, \varepsilon_{2}>0$. Пусть также $d \geqslant 4$,

$$
B \in C_{\left[\tau_{1}, \theta_{1}\right]}^{d}\left((\mathscr{B} \cup D)^{\langle d, 1\rangle} \mid \mathscr{B}_{\langle 1,1\rangle}^{\langle d, 1\rangle}\right) \cap C_{\left[\tau_{2}, \theta_{2}\right]}^{d}\left((\mathscr{B} \cup D)^{\langle d, 1\rangle} \mid \mathscr{D}_{\langle 1,1\rangle}^{\langle d, 1\rangle}\right) .
$$

Тогда при достаточно больших $\varkappa$

$$
\alpha^{1}\left((\mathscr{B} \cup D)_{B}^{(2)}\right)=\left(1+O\left(2^{-\log ^{2} x}\right)\right) \alpha^{1}\left((\mathscr{B} \cup D)_{[3]}^{(1,2)}\right) .
$$


Доказательство. Доказательство аналогично доказательству леммы 13. Заметим, что

$$
\alpha^{1}(\mathscr{B} \cup D)=\alpha^{1}(\mathscr{B})+\alpha^{1}(\mathscr{D})=\alpha^{1, d}\left(\mathscr{B}_{\langle 1,1\rangle}^{\langle d, 1\rangle}\right)+\alpha^{1, d}\left(\mathscr{D}_{\langle 1,1\rangle}^{\langle d, 1\rangle}\right) .
$$

Положим $B=B_{1} \cup B_{2}$, где $B_{1} \in C_{\left[\tau_{1}, \theta_{1}\right]}^{d}\left(\mathscr{B}_{\langle 1,1\rangle}^{\langle d, 1\rangle}\right), B_{2} \in C_{\left[\tau_{2}, \theta_{2}\right]}^{d}\left(\mathscr{D}_{\langle 1,1\rangle}^{\langle d, 1\rangle}\right)$. Аналогично (51) и (52), из взаимной регулярности $\mathscr{B}$ и $\mathscr{D}$ для произвольных $\left\{v_{0}\right\} \in \mathscr{B},\left\{u_{0}\right\} \in \mathscr{D}$ следует, что

$$
\begin{aligned}
\alpha^{1}\left((\mathscr{B} \cup D)_{B,[2]}^{(2)}\right)= & \sum_{\{v\} \subseteq B_{1}} \alpha^{1}\left((\mathscr{B} \cup D)_{\{v\},[2]}^{(2)}\right)+\sum_{\{u\} \subseteq B_{2}} \alpha^{1}\left((\mathscr{B} \cup D)_{\{u\},[2]}^{(2)}\right) \\
= & \left(1+O\left(2^{-\log ^{2} x}\right)\right)\left(\left|B_{1}\right| \alpha^{1}\left((\mathscr{B} \cup D)_{\left\{v_{0}\right\},[2]}^{(2)}\right)+\left|B_{2}\right| \alpha^{1}\left((\mathscr{B} \cup D)_{\left\{u_{0}\right\},[2]}^{(2)}\right)\right) \\
= & \left(1+O\left(2^{-\log ^{2} x}\right)\right)\left(\alpha^{1}(\mathscr{B}) \alpha^{1}\left((\mathscr{B} \cup D)_{\left\{v_{0}\right\},[2]}^{(2)}\right)+\alpha^{1}(\mathscr{D}) \alpha^{1}\left((\mathscr{B} \cup D)_{\left\{v_{0}\right\},[2]}^{(2)}\right)\right) \\
= & \left(\sum_{\{v\} \in \mathscr{B}} f(\{v\}) \alpha^{1}\left((\mathscr{B} \cup D)_{\{v\},[2]}^{(2)}\right)+\sum_{\{u\} \in \mathscr{D}} f(\{u\}) \alpha^{1}\left((\mathscr{B} \cup D)_{\{u\},[2]}^{(2)}\right)\right) \\
& \times\left(1+O\left(2^{-\log ^{2} x}\right)\right)=\alpha^{1}\left((\mathscr{B} \cup D)_{[3]}^{(1,2)}\right)\left(1+O\left(2^{-\log ^{2} x}\right)\right) .
\end{aligned}
$$

Лемма доказана.

Теорема 10. Пусть $I=(X, f)$ является $(3, \varkappa, q, c)$-ординарной функциональной парой, семейства $\mathscr{B} \subseteq \mathscr{A}_{\hat{3}}(I)$ и $\mathscr{D} \subseteq \mathscr{A}_{\hat{3}}(I)$ являются взаимно регулярными. Тогда

$$
S(\mathscr{B} \cup D)=\left(1+O\left(2^{-(1 / 2) \log ^{2} \varkappa}\right)\right) \exp \left\{\hat{\mu}_{3}(\mathscr{B} \cup D)\right\}
$$

Утверждение вытекает из следствия 4, теоремы 8 и леммы 14.

\section{Список литературы}

1. Сапоженко А. А., О числе антицепей в ранжированных частично упорядоченных множествах. Дискретная математика (1989) 1, №1, 74-93.

2. Сапоженко А. А., О числе антицепей в многослойных ранжированных множествах. Дискретная математика (1989) 1, №2, 110-128.

3. Сапоженко А. А., О предельных распределениях случайных величин, порожденных ограниченными последовательностями. Дискретный анализ (1994) 27, 166-176.

4. Сапоженко А. А., Проблема Дедекинда и метод граничных функционалов. Maтем. вопросы кибернетики (2000) 9, 161-220.

Статья поступила 10.11.2003. 